\title{
Renal denervation therapy for hypertension: truths and half-truths
}

\author{
C. Venkata S. Ram ${ }^{1,2 *}$, MD; S.S. Iyengar ${ }^{3}$, MD; Gurpreet S. Wander ${ }^{4}$, MD; Tiny Nair ${ }^{5}$, MD; \\ A. Sreenivas Kumar ${ }^{1}, \mathrm{MD}$; Saumitra $\mathrm{Ray}^{6}$, MD; Satyavan Sharma ${ }^{7}, \mathrm{MD}$; \\ on behalf of the Hypertension Council, Cardiological Society of India
}

1. Apollo Medical College, Hyderabad, Telangana, India; 2. University of Texas Southwestern Medical Center, Dallas, TX, USA;

3. Manipal Hospital, Bangalore, India; 4. Hero DMC Heart Institute, Dayanand Medical College and Hospital, Ludhiana, India;

5. PRS Hospital, Killipalam, Thiruvananthapuram, India; 6. Woodland Hospital, BM Birla Heart Centre, Kolkata, West Bengal,

India; 7. Bombay Hospital \& Medical Research Centre, Mumbai, India

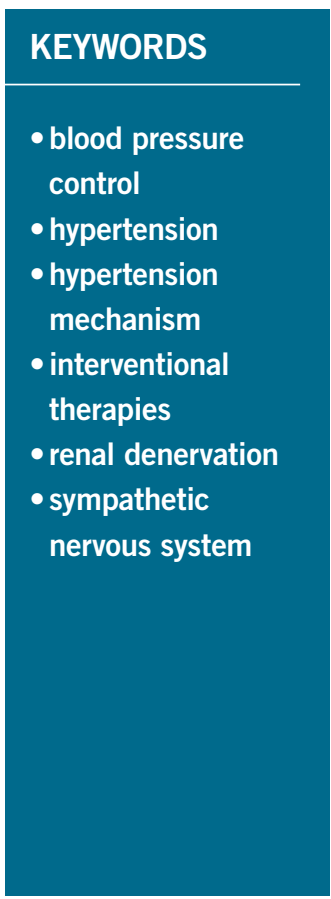

\section{Abstract}

Systemic hypertension is a major contributing factor for excessive morbidity and mortality globally. Experimental studies and early clinical trials showed excellent therapeutic responses to renal denervation (RDN) in patients with hypertension. However meta-analyses and objective assessments have failed to show that RDN therapy has any significant effect on blood pressure. The aim of this review is to introduce the different methods that can be used in RDN, along with the benefits and disadvantages of these methods. Radiofrequency (RF) ablation (of renal nerves) is the most common method of RDN, and we discuss the clinical evaluation of this method in the SYMPLICITY RDN trials. Finally, the development of secondgeneration RF devices and more comprehensive RDN procedures lead us to consider the current status and future path for RDN.

\footnotetext{
*Corresponding author: University of Texas Southwestern Medical Center, 5323 Harry Hines Blvd, Dallas, TX 75390, USA. E-mail:drram_v@apollohospitals.com
} 


\section{Introduction}

Systemic hypertension affects a large percentage of the adult population worldwide. It is a powerful risk factor for cardiovascular disease (CVD), cerebrovascular disease (CeVD), and chronic kidney disease (CKD). Uncontrolled hypertension causes considerable morbidity and excessive mortality ${ }^{1}$. Blood pressure control with pharmacological therapy has been shown to prevent hypertension-related complications and prolong longevity. Despite the proven success of drug therapy, hypertension remains poorly controlled in the community due to multi-factorial non-adherence. Also, in some, hypertension remains "resistant" to adequate therapy. To offset these difficulties in the real world, renal denervation (RDN) has been developed (Figure 1). Experimental and clinical studies ${ }^{2,3}$ have shown that RDN lowers blood pressure in hypertension. The development of RDN to treat hypertension was welcomed as a "spectacular" therapeutic breakthrough in medical science. The potential application of RDN in clinical practice created a breathtaking frenzy, triggering publicity never witnessed before in clinical medicine. Research interest and scientific progress in the utility of RDN therapy have advanced rapidly. However, the enthusiasm for this technical marvel subsided as quickly as its initial ascent to fame.

\section{RATIONALE FOR THE TREATMENT OF HYPERTENSION WITH RDN}

The pathogenesis of hypertension is multifactorial and complex but the sympathetic nervous system (SNS) plays an important aetiological role in elevating the blood pressure and heart rate. Thus, interruption of the SNS is an established pharmacotherapy for hypertension but is fraught with adverse effects. To eliminate this hindrance, RDN (as a non-pharmacological alternative) appears to have potential to treat hypertension. Surgical sympathectomy in the 1950s was shown to offer remarkable benefits in the treatment of hypertension. However, this procedure was discontinued due to intolerable adverse effects such as postural hypotension. RDN, however, is a more selective method compared to crude surgical sympathectomy and has a strong experimental foundation. It is known that stimulation of renal sympathetic efferent nerves lowers the blood pressure by reversing the pathophysiological mechanisms of hypertension. Elegant studies using radiofrequency (or other means of) ablation of renal nerves have shown a reduction in systemic blood pressure by lowering the SNS tone. Reduction of systemic vascular resistance is the reason for the antihypertensive effect of RDN. Thus, from an experimental bench to clinical bedside, RDN is a valid therapeutic tool to lower blood pressure.

\section{METHODS USED FOR RENAL DENERVATION}

Radiofrequency (RF) ablation (of renal nerves) is the most common method of RDN. RF ablation causes tissue damage and "denervation" through thermal injury to the renal artery. Both the Symplicity (Medtronic, Minneapolis, MN, USA) and EnligHTN'M (Abbott Vascular, Santa Clara, CA, USA) catheters cause RF ablation of the renal nerves. Several advances in techniques and procedures have been developed to refine RDN - all using the RF method. Over the years, RF for RDN has matured and has been applied extensively in experimental and clinical settings.

\section{ULTRASOUND-BASED RDN}

High-frequency ultrasound (US) has been applied to cause selective RDN. Experimental and clinical studies validated the potential utility of US-mediated RDN therapy. Selective renal injury and a reduction of SNS tone have been demonstrated with US. Preliminary studies with US have shown promising results ${ }^{2}$. As in RF, US technology has advanced to preserve the vascular

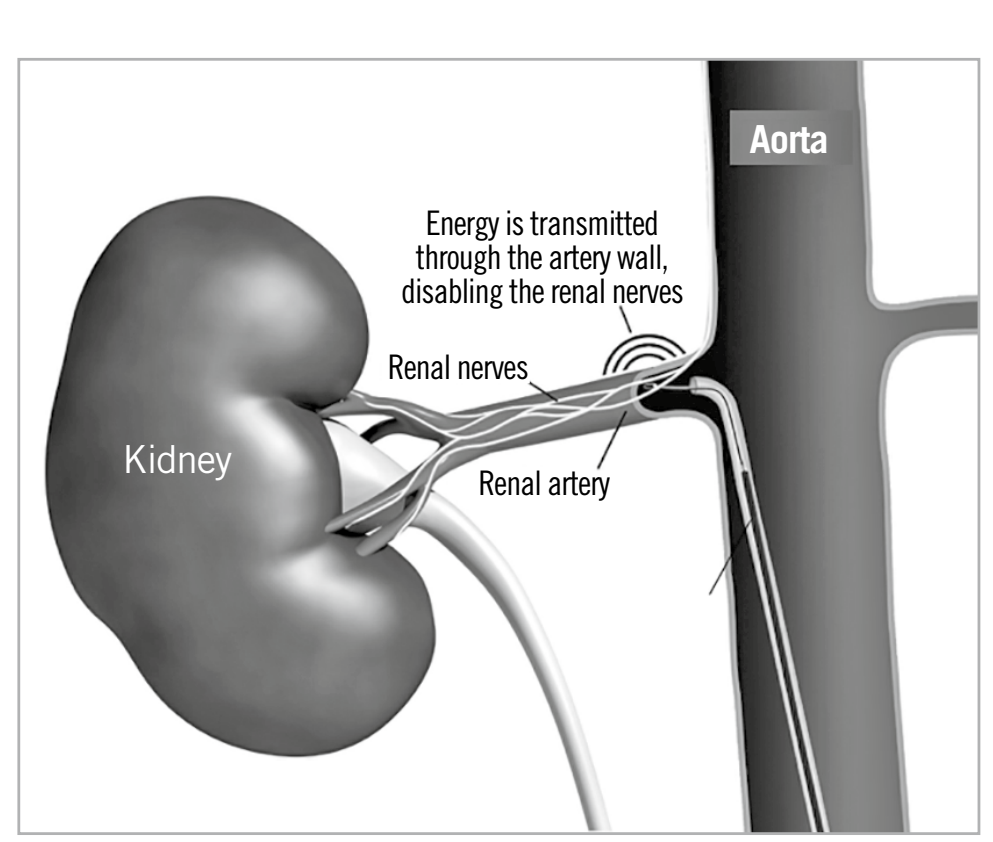

Figure 1. The concept of catheter-based renal denervation. 
endothelium irrespective of the renal artery size and morphology. This technique could be difficult in some patients. Whether US-mediated RDN is superior to RF ablation is a matter of ongoing debate and clinical studies.

\section{CHEMICAL RENAL DENERVATION}

Chemical denervation causes renal neurolysis and reduces the SNS tone and blood pressure in animal models. Selective injury to the renal nerves by alcohol, vincristine, and guanethidine leads to renal nerve demyelination. While chemical denervation has a biophysiological rationale, there is no clinical evidence to date about its feasibility for clinical hypertension. In comparison to RF ablation, chemical denervation induces deeper lesions and a significant reduction in SNS traffic. While chemical denervation is promising, a thorough and comprehensive evaluation of its safety should be ascertained.

\section{CLINICAL OUTCOMES FROM THE EARLIER RDN SYMPLICITY TRIALS}

The clinical evaluation of RF ablation for RDN began with the application of the first-generation Symplicity catheter system. A single unipolar electrode was utilised to induce RDN by rotating and navigating the catheter through the renal arteries in a helical template. Symplicity HTN-1 was the very first clinical study (45 patients) to test RDN in patients with hypertension ${ }^{2,3}$. The study showed a marked fall in the office blood pressure levels which was sustained for up to 36 months. In a subset of subjects, a reduction of norepinephrine content after RDN was noted, confirming the underlying pathophysiological concept.

In the Symplicity HTN-2 trial, patients with "resistant" hypertension underwent $\mathrm{RDN}^{4}$. A substantial improvement in the office blood pressure levels and 24-hr ambulatory systolic blood pressure level was reported (Figure 2). In this unblinded study, the majority of the patients responded to RDN therapy. In both the Symplicity HTN-1 and 2 trials, the results were based on office blood pressure measurement and not on ambulatory or home blood pressure recordings. The lack of sham control in both of the studies was widely criticised.

The SYMPLICITY HTN-3 trial was a crucial clinical research study in which a "sham" control group was included and 24-hr ambulatory blood pressure measurement was mandated in the study groups ${ }^{5}$. At six months, there was no significant difference in the blood pressure levels of RDN-treated and sham patients. Thus, it showed that RDN was not superior to placebo in the treatment of resistant hypertension. The fall in blood pressure of sham patients was substantial. The results of the SYMPLICITY HTN-3 trial nearly disqualified RDN as a therapeutic option to treat hypertension. Various explanations (incomplete denervation, lack of operator experience, etc.) were offered for the failure of RDN therapy in the SYMPLICITY HTN-3 trial.

\section{RDN THERAPY AFTER THE SYMPLICITY TRIALS}

After the failure of RDN in the SYMPLICITY HTN-3 trial, there was a revitalised effort to improve the effectiveness of the technique by modifying the method and catheter technology, and considering the renal nerve anatomy. These revised principles have resurrected RDN to some extent. By developing the second-generation RF catheters, a more complete and effective RDN was made possible. Thus, a more comprehensive RDN procedure was developed to cause denervation of the main and branch renal arteries. To overcome the shortcomings of the earlier Symplicity trials, blinded sham-controlled trials - SPYRAL HTN-OFF MED and SPYRAL

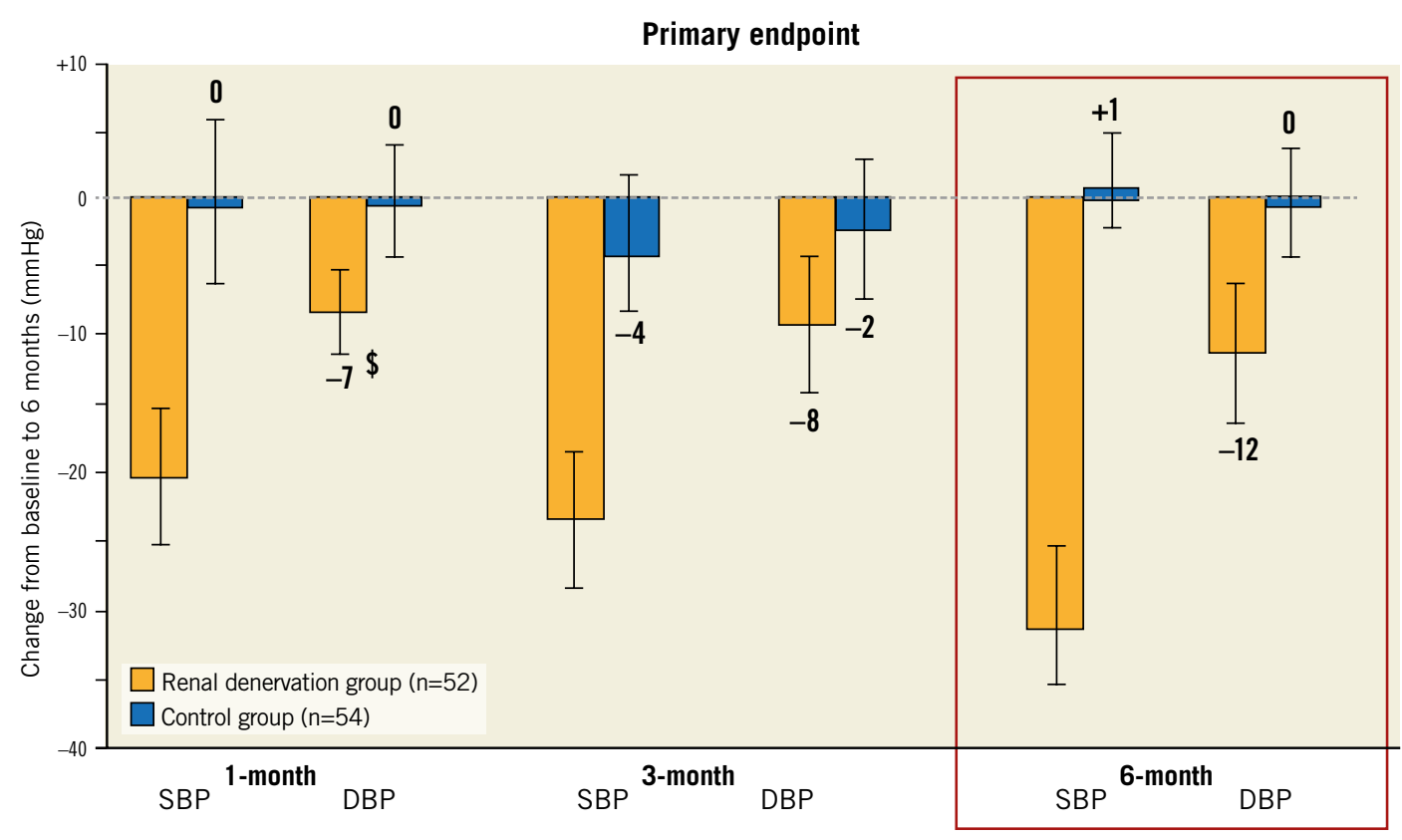

Figure 2. Symplicity HTN-2: uncontrolled study. 
HTN-ON MED - were carefully designed, and completed ${ }^{6,7}$. Both these studies evaluated the 24-hr ambulatory blood pressure response to RDN in patients with mild-moderate hypertension. In the "OFF" protocols, patients were not on antihypertensive drugs at the time of the enrolment, whereas they were on 1-3 antihypertensive drugs in the "ON" study at the time of enrolment. Both studies showed significant blood pressure lowering effects from RDN at six months (Figure 3). The antihypertensive effects of RDN, while small, were significant in both the studies. These observations have rekindled the potential usefulness of RDN to some extent by the newer catheter technology and patient selection criteria. It should be noted that the blood pressure fall in these newer studies was at best modest, not substantial by any standard.

The RADIANCE-HTN SOLO trial evaluated the efficacy of US-mediated RDN compared to sham controls in patients with

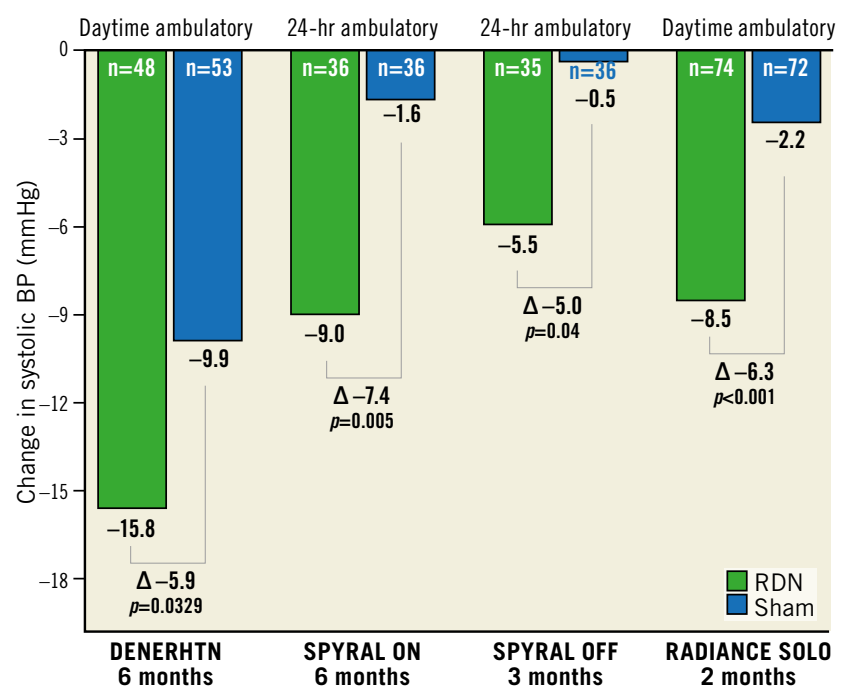

Figure 3. Summary of recent randomised trials on renal denervation. mild-moderate hypertension who were off antihypertensive drugs for at least four weeks before the enrolment. At two months after the procedure, the blood pressure fall was significant in the RDN group compared to the sham group. The fall in 24-hr ambulatory blood pressure level was significant in the RDN group. The degree of 24-hr blood pressure reduction was similar in the SPYRAL-HTN and RADIANCE-HTN SOLO studies, indicating that a clarified approach with refinements in procedural techniques and patient selection may indeed be the decisive factors in responses to RDN. The recently published Global SYMPLICITY Registry (GSR) results infuse some enthusiasm for the revival of RDN in hypertension ${ }^{8}$. The data from 3,000 subjects from 196 centres/45 countries suggest that RDN performed diligently in "high-risk" groups is effective in lowering the blood pressure in patients followed up to three years (Figure 4). All the patients had "uncontrolled" hypertension and comorbidities when the SNS was activated - diabetes, CKD, congestive heart failure, arrhythmias, and obstructive sleep apnoea. Blood pressure reduction was similar in all the "high-risk" cohorts and in those with a propensity to premature CVD. The office blood pressure lowering effect was greater than ambulatory blood pressure levels. In some patients, the blood pressure fall was impressive, while in others it was modest. Meta-analyses have suggested that RDN therapy does not produce significant effects on blood pressure in patients with hypertension (Figure 5, Figure 6).

\section{CURRENT STATUS AND FUTURE PATH FOR RDN}

The concept of sympatholytic consequences of RDN therapy in lowering the blood pressure is based on the pathophysiology of hypertension. Systemic hypertension is a heterogeneous entity with wide variations in response to any therapeutic intervention. So, RDN therapy is not an exception. The initial proof-of-concept clinical experience with RDN demonstrated dramatic therapeutic results. RDN was welcomed as the best tonic to solve the perplexity

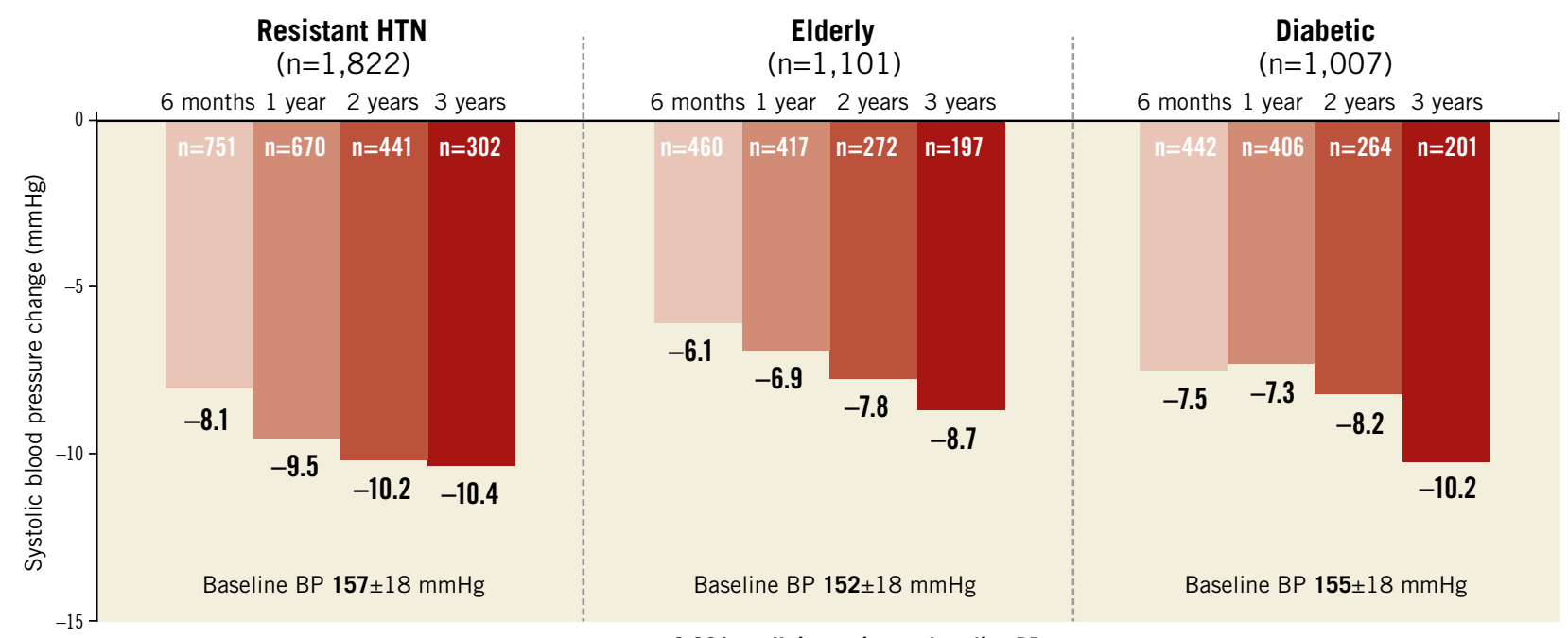

$p<0.001$ at all time points vs baseline BP

Figure 4. Global RDN Registry: 24-hr systolic BP change. 


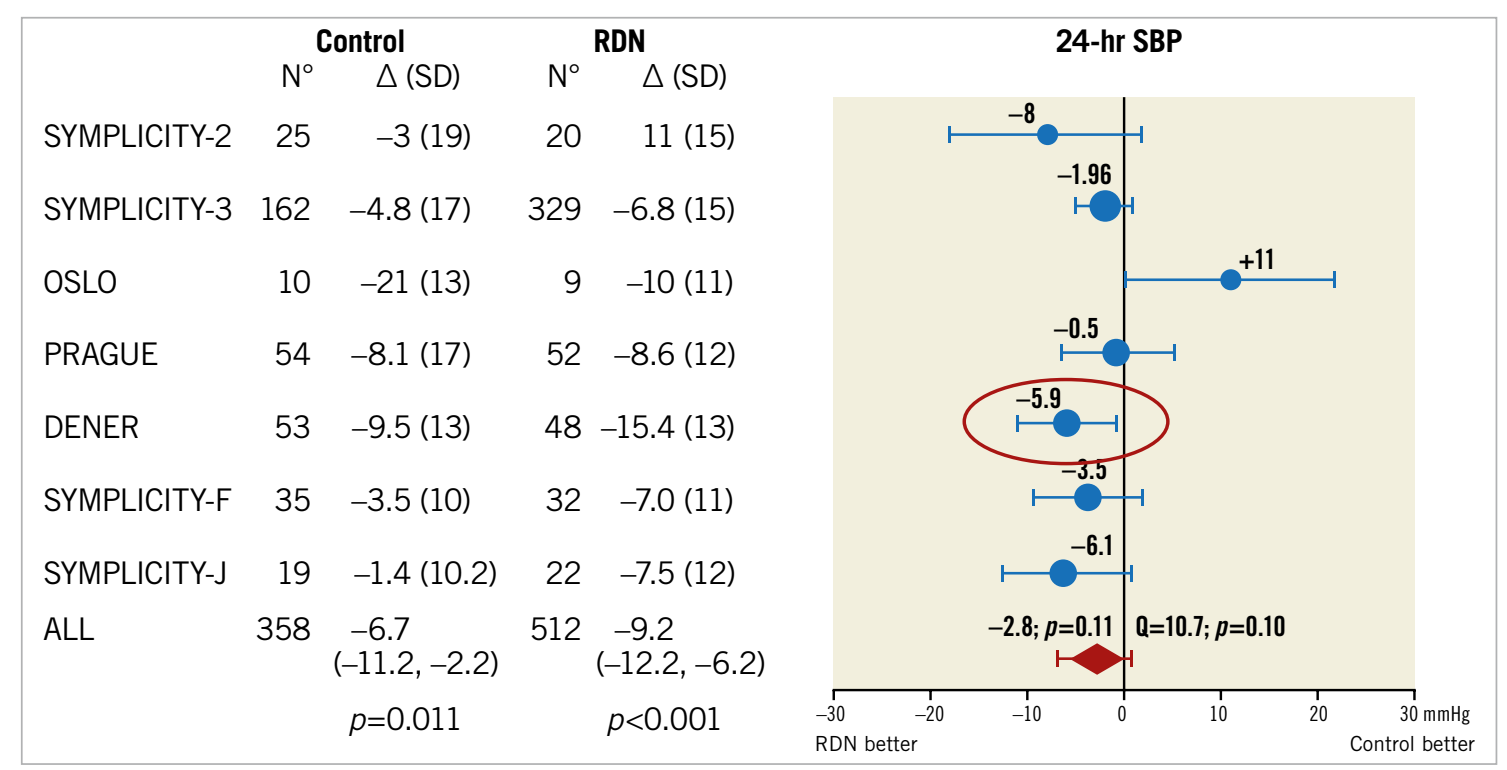

Figure 5. Meta-analysis of randomised controlled trials of renal denervation in treatment-resistant hypertension showing 24-hr systolic blood pressure.

of hypertension. The initial uncontrolled studies showed significant drops in blood pressure level by as much as $32 / 20 \mathrm{mmHg}$ lasting up to 36 months! The spirited ardour for RDN faded suddenly when the sham-controlled randomised SYMPLICITY HTN-3 concluded that RDN was not superior to sham (drug therapy). The publication of SYMPLICITY HTN-3 results instantly destabilised the leverage that RDN had enjoyed previously. The RDN pathway to treat hypertension, therefore, decelerated rather quickly. Lack of rigorous control groups, changes in treatment, non-compliance, and procedural failures/insufficiencies have cast much doubt on the role of RDN for the treatment of hypertension.
With the improvements in catheter technology and "resurrection" of the mechanical procedures to accomplish effective and near-complete denervation, there appears to be cautious optimism in RDN therapy. The most recent "post-Symplicity" trials indicate that RDN therapy may indeed be a valid concept worthy of further testing. Taken together, the post-Symplicity studies show that the average blood pressure reduction with RDN is at best modest, probably equivalent to taking one antihypertensive drug. In nearly one third of patients subjected to RDN therapy, there is no change in the blood pressure level at all, and in some patients the blood pressure may go up! How can we reconcile

\begin{tabular}{|c|c|c|c|c|c|c|c|c|}
\hline \multirow[b]{3}{*}{ SYMPLICITY-2 } & \multicolumn{2}{|c|}{ Control } & \multicolumn{2}{|r|}{ RDN } & \multirow{2}{*}{\multicolumn{4}{|c|}{ Office SBP }} \\
\hline & $\mathrm{N}^{\circ}$ & $\Delta(\mathrm{SD})$ & $\mathrm{N}^{\circ}$ & $\Delta(\mathrm{SD})$ & & & & \\
\hline & 51 & $+1.0(21)$ & 49 & $-32(23)$ & 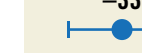 & & & \\
\hline SYMPLICITY-3 & 171 & $-11.7(26)$ & 353 & $-14.1(24)$ & & & & \\
\hline OSLO & 10 & $-28(13)$ & 9 & $-8(15)$ & & & +20 & \\
\hline PRAGUE & 54 & $-14.3(20)$ & 52 & $-12.4(17)$ & & $\vdash$ & +1.9 & \\
\hline DENER & 53 & $-9.5(20)$ & 48 & $-15.1(20)$ & & -5.6 & & \\
\hline SYMPLICITY-J & 19 & $-7.9(21)$ & 22 & -16.6 (19) & & -8.7 & -1 & \\
\hline ALL & 358 & $\begin{array}{l}-11.6 \\
(-20.3,-2.8)\end{array}$ & 533 & $\begin{array}{l}-16.5 \\
(-24.0,-9.0)\end{array}$ & & $-4.9 ; p=0.47$ & $Q=60.5 ; p<0.001$ & \\
\hline & & $p=0.019$ & & $p=0.002$ & $\begin{array}{l}-50 \\
\text { RDN better }\end{array}$ & 25 & 25 & $\begin{array}{r}50 \mathrm{mmHg} \\
\text { Control better }\end{array}$ \\
\hline
\end{tabular}

Figure 6. Meta-analysis of randomised controlled trials of renal denervation in treatment-resistant hypertension showing office systolic blood pressure. 
these disparate results? The question has always been whether responders to RDN were non-compliant with medical therapy previously. Thus, to give RDN a realistic chance, patient adherence to antihypertensive drug therapy should be assured. It is unclear why women seem to respond to RDN to a greater extent than men. Are they more compliant with drug therapy after the RDN procedure?

We must acknowledge that, even in the best operational hands, the blood pressure response appears to be very modest (3-6 mm drop in systolic blood pressure). The enthusiasts who earlier hailed RDN therapy as producing dramatic results have now compromised to accept a modest response to $\mathrm{RDN}^{9}$. There is no longer much talk about "hyper-responders"; instead, we now settle for "responders"! The challenge remains how to identify responders from non-responders. Until this transpires, we will continue to witness a mixed bag of responders and non-responders to RDN therapy. Selection of the right candidates who might benefit from RDN is of fundamental importance to decide the value of RDN therapy. There are those patients who demonstrate a dramatic therapeutic response, and we need to "spot" them. But how? Not as easy as spotting a zebra among horses!

We have improved the catheter technology to deliver optimal mechanical RDN since the SYMPLICITY HTN-3 results. So, we can divide the RDN story into "pre-SYMPLICITY" and "postSYMPLICITY" time periods. Yet, the procedure is not ready for its heyday! The best results can be expected only from centres with technical expertise. How can we apply these observations to a clinical condition as common as hypertension? If the outcome is operator dependent, the scope of RDN will be very narrow in community practice. Even with direct surgical sympathectomy, a significant reduction in blood pressure was seen in only $27 \%$ of patients. So, it is unrealistic to expect predictable responses to an indirect procedure such as RDN. A main hurdle with RDN therapy is that the procedure has not been standardised like other cardiovascular interventional procedures. Hence, it is impossible to estimate in each patient whether RDN in fact causes denervation. We need to find out the measures or markers of effective denervation; otherwise, it is like shooting in the dark. Even before establishing the benchmarks for effective denervation in hypertension, the procedure drifted into areas such as obstructive sleep apnoea, $\mathrm{CKD}$, congestive heart failure, and atrial fibrillation, etc., thus losing focus and direction, resulting in the present state of uncertainty and clinical disarray.

From the physiological and pathophysiological mechanisms, interruption of the SNS makes sense. The question is whether RDN can induce sufficient blood pressure reduction in patients with hypertension. Is it possible to denervate selectively only the vasopressor renal artery fibres and spare the vasodepressor fibres? Otherwise, denervation of vasodepressor nerve fibres may paradoxically raise the blood pressure level. Future research models should pinpoint denervation of only the vasopressor nerve traffic. Non-selective and crude denervation by the current methodology may explain the inconsistent therapeutic responses to RDN.
After years of dedicated research, RDN therapy has come of age. The procedure has seen many ups and downs in its development. RDN experience has gone through three evolutionary phases - rapid rise, steep fall, and gradual resurrection. The gruelling challenge is to identify the subsets of hypertensive patients who are likely to respond to therapy. The post-Symplicity trials have shown a ray of hope for RDN therapy after the gloom cast by the results of the SYMPLICITY HTN-3 trial and various metaanalyses. So, the question remains - will the clouds pass to allow some light to fall on the future role of RDN in the treatment of hypertension? Until then, we need to keep our eyes open, gazing at the beautiful sky.

\section{Acknowledgements}

The authors thank Ms N. Madhavi Latha for her editorial assistance and for transcribing this paper.

\section{Conflict of interest statement}

The authors have no conflicts of interest to declare.

\section{References}

1. Whelton PK, Carey RM, Aronow WS, Casey DE Jr, Collins KJ, Dennison Himmelfarb C, DePalma SM, Gidding S, Jamerson KA, Jones DW, MacLaughlin EJ, Muntner P, Ovbiagele B, Smith SC Jr, Spencer CC, Stafford RS, Taler SJ, Thomas RJ, Williams KA Sr, Williamson JD, Wright JT Jr. 2017 ACC/AHA/AAPA/ABC/ ACPM/AGS/APhA/ASH/ASPC/NMA/PCNA Guideline for the Prevention, Detection, Evaluation, and Management of High Blood Pressure in Adults: Executive Summary: A Report of the American Heart Association Task Force on Clinical Practice Guidelines. J Am Coll Cardiol. 2018;71:2199-269.

2. Azizi M, Schmieder RE, Mahfoud F, Weber MA, Daemen J, Davies J, Basile J, Kirtane AJ, Wang Y, Lobo MD, Saxena M, Feyz L, Rader F, Lurz P, Sayer J, Sapoval M, Levy T, Sanghvi K, Abraham J, Sharp ASP, Fisher NDL, Bloch MJ, Reeve-Stoffer H, Coleman L, Mullin C, Mauri L; RADIANCE-HTN Investigators. Endovascular ultrasound renal denervation to treat hypertension (RADIANCE-HTN SOLO): a multicentre, international, singleblind, randomised, sham-controlled trial. Lancet. 2018;391: 2335-45

3. Krum H, Schlaich MP, Sobotka PA, Böhm M, Mahfoud F, Rocha-Singh K, Katholi R, Esler MD. Percutaneous renal denervation in patients with treatment-resistant hypertension: final 3-year report of the Symplicity HTN-1 study. Lancet. 2014;383: 622-9.

4. Esler MD, Böhm M, Sievert H, Rump CL, Schmieder RE, Krum H, Mahfoud F, Schlaich MP. Catheter-based renal denervation for treatment of patients with treatment-resistant hypertension: 36 month results from the SYMPLICITY HTN-2 randomized clinical trial. Eur Heart J. 2014;35:1752-9.

5. Bhatt DL, Kandzari DE, O’Neill WW, D’Agostino R, Flack JM, Katzen BT, Leon MB, Liu M, Mauri L, Negoita M, Cohen SA, Oparil S, Rocha-Singh K, Townsend RR, Bakris GL; SYMPLICITY 
HTN-3 Investigators. A controlled trial of renal denervation for resistant hypertension. N Engl J Med. 2014;370:1393-401.

6. Townsend RR, Mahfoud F, Kandzari DE, Kario K, Pocock S, Weber MA, Ewen S, Tsioufis K, Tousoulis D, Sharp ASP, Watkinson AF, Schmieder RE, Schmid A, Choi JW, East C, Walton A, Hopper I, Cohen DL, Wilensky R, Lee DP, Ma A, Devireddy CM, Lea JP, Lurz PC, Fengler K, Davies J, Chapman N, Cohen SA, DeBruin V, Fahy M, Jones DE, Rothman M, Böhm M; SPYRAL HTN-OFF MED trial investigators. Catheter-based renal denervation in patients with uncontrolled hypertension in the absence of antihypertensive medications (SPYRAL HTN-OFF MED): a randomised, sham-controlled, proof-of-concept trial. Lancet. 2017;390:2160-70.
7. Kandzari DE, Böhm M, Mahfoud F, Townsend RR, Weber MA, Pocock S, Tsioufis K, Tousoulis D, Choi JW, East C, Brar S, Cohen SA, Fahy M, Pilcher G, Kario K; SPYRAL HTN-ON MED Trial Investigators. Effect of renal denervation on blood pressure in the presence of antihypertensive drugs: 6-month efficacy and safety results from the SPYRAL HTN-ON MED proof-of-concept randomised trial. Lancet. 2018;391:2346-55.

8. Mahfoud F, Mancia G, Schmieder R, Narkiewicz K, Ruilope L, Schlaich M, Whitbourn R, Zirlik A, Zeller T, Stawowy P, Cohen SA, Fahy M, Böhm M. Renal denervation in high-risk patients with hypertension. J Am Coll Cardiol. 2020;75:2879-88.

9. Ram CVS. Status of Renal Denervation Therapy for Hypertension. Circulation. 2019;139:601-3. 